\title{
Thrombocytopenia with congenital dyserythropoietic anemia
}

INSERM

\section{Source}

INSERM. (1999). Orphanet: an online rare disease and orphan drug data base.

Thrombocytopenia with congenital dyserythropoietic anemia. ORPHA:67044

Thrombocytopenia with congenital dyserythropoietic anemia (CDA; see this term) is a rare hematological disorder, seen almost exclusively in males, characterized by moderate to severe thrombocytopenia with hemorrhages with or without the presence of mild to severe anemia. 